InnOvaciOnes de NegOciOs 16(31): 87-117

(c) 2019 UANL, Impreso en México (ISSN: 2007-1191)

Recepción: 9 Diciembre de 2018. Aceptación: 12 Diciembre 2018

\title{
Las remuneraciones impactan positivamente en el compromiso organizacional mediante el empowerment psicológico en docentes de instituciones públicas (The remunerations positively impact on the organizational commitment through the psychological empowerment in public institutions teachers)
}

\author{
Rosalba Treviño Reyes, Adriana Segovia Romo, Elías Alvarado Lagunas, \\ Pablo Guerra Rodríguez \\ Universidad Autónoma de Nuevo León \\ rosalba.trevinorys@uanl.edu.mx, adrianasegovia@hotmail.com, \\ eliaxalvarado@gmail.com, pablo.guerrard@uanl.edu.mx
}

\begin{abstract}
Currently, a worrying reality is being experienced, since $79 \%$ of the employees in each company are not committed to their work. Organizations today are trying to achieve $100 \%$ of their results in a highly competitive environment, full of threats and obstacles, where the economic compensation is no longer the most important aspect for employees. With the economic and social changes that are currently experienced, organizations now not only worry about achieving competitiveness and excellent results, but they also face new challenges related to human capital. The present investigation analyzes the relation between remunerations, the psychological empowerment and the organizational commitment in teachers of public institutions of middle higher education. In addition to this, the current context of the problem is emphasized, the literature review, the methodology that was followed through the development of the measurement instrument, the content validity and the data collection that were analyzed under a mediation model. A model of structural equations was used as the method of analysis. It was found that the results support the positive relationship between compensation and organizational commitment through psychological empowerment, so that the hypothesis is accepted.
\end{abstract}

Las remuneraciones impactan positivamente en el compromiso 
Key words: human capital, organizational commitment, remunerations, psychological empowerment, teachers.

JEL : M12, M1.

Resumen. Actualmente se vive una realidad preocupante, ya que el $79 \%$ de los empleados en cada empresa no están comprometidos con su trabajo. Las organizaciones de hoy están tratando de conseguir el $100 \%$ de sus resultados en un entorno altamente competitivo, lleno de amenazas y obstáculos, donde la remuneración económica dejó de ser el aspecto más importante para los empleados. Con los cambios económicos y sociales que se viven actualmente, las organizaciones ahora no sólo se preocupan por lograr la competitividad y excelentes resultados, sino que enfrentan nuevos desafíos relacionados con el capital humano. La presente investigación analiza la relación entre las remuneraciones, el empowerment psicológico y el compromiso organizacional en docentes de instituciones públicas de educación media superior. Aunado a ello se enfatiza en el contexto actual del problema, la revisión de literatura, la metodología que se siguió a través de la elaboración del instrumento de medición, la validez de contenido y la recolección de datos que se analizaron bajo un modelo de mediación. Se utilizó un modelo de ecuaciones estructurales como método de análisis. Se encontró que los resultados avalan la relación positiva entre las remuneraciones y el compromiso organizacional mediante el empowerment psicológico, por lo que se acepta la hipótesis planteada.

Palabras clave: capital humano, compromiso organizacional, remuneraciones, empowerment psicológico, docentes

\section{Introducción}

De acuerdo con los cambios económicos y sociales que se viven actualmente, las organizaciones ahora no sólo se preocupan por lograr la competitividad y excelentes resultados, sino que enfrentan nuevos

Treviño, R., Segovia, A., Alvarado, E., Guerra, P. 
desafíos relacionados con el capital humano. Hoy en día, el trabajo necesita tener un sentido especial para las personas, considerando factores como la calidad de vida y el crecimiento personal para tomar la decisión de permanecer o no en su área laoral. Cuando esto no sucede en el empleo, las condiciones laborales se tornan complicadas y emergen diversas problemáticas que llegan a afectar a las organizaciones, pero sobre todo a su fuerza laboral.

La integración de las personas a la organización es un asunto de vital importancia, pues de este acoplamiento depende en gran medida que los intereses, objetivos y necesidades de cada uno lleguen a un nivel armónico que permitan que la relación empleado-empresa perdure en el tiempo. Desde la perspectiva de los empleados, el compromiso organizacional permite estabilidad laboral, que impacta en prestaciones, jubilación, beneficios sociales, y garantiza cubrir necesidades tanto materiales como psicológicas (reconocimiento, desarrollo de habilidades, socialización, estatus) beneficiando a las familias y economía del país (Betanzos y Paz, 2007).

Para los investigadores y personal de recursos humanos la importancia del compromiso organizacional se debe principalmente a que tiene un impacto directo en actitudes y conductas del empleado; como aceptación de metas, valores y cultura de la organización, menor ausentismo y baja rotación de personal entre otros. Por ello es un concepto que ha crecido en importancia en la psicología organizacional, y se centra en estudiar los vínculos que se generan entre los empleados y la organización (Betanzos y Paz, 2007).

\section{Las remuneraciones impactan positivamente en el compromiso}


Retomando un importante trabajo del contexto actual organizacional, se observa que en este estudio se obtuvieron 7,700 encuestas a millennials de 29 países de mercados emergentes y desarrollados alrededor del mundo en 2015, los resultados arrojaron que dos tercios de ellos expresan su sentimiento de querer dejar su organización para el 2020 (Deloitte, 2016). Esto lleva a pensar que si no hay cambios en la manera de retener y preparar talento, las empresas en México enfrentarán problemas en la incorporación de la generación millennial y en la escasez de perfiles competitivos, problema que hoy afecta a 4 de cada 10 organizaciones en el país (Cadavid, 2015, citado en CNNExpansión, 2016). Otro estudio más reciente de Deloitte (2017), su quinta encuesta global sobre millennials, indica que el $66 \%$ prefiere esquemas flexibles de trabajo para aumentar el compromiso, la salud, la felicidad y alcanzar los objetivos de la organización.

En la actualidad, las condiciones laborales determinan estados psicológicos positivos y bienestar laboral. En México, las organizaciones se caracterizan por otorgar recompensas insuficientes a sus miembros, como lo indica el hecho de que este país no ocupa los primeros lugares en un ranking mundial de salarios promedio. Puede decirse entonces que los trabajadores mexicanos desarrollan sus actividades en un ambiente precario, que limita su posibilidad de identificarse con la organización y adaptarse al rol de trabajo, disminuyendo su productividad y la obtención de satisfacción laboral (Calderón, Laca, Pando y Pedroza, 2015).

Un estudio realizado por Gallup en 2018 identificó a tres tipos de empleados en las organizaciones:

Treviño, R., Segovia, A., Alvarado, E., Guerra, P. 
a) Los empleados comprometidos: es decir, que trabajan con pasión y tienen una profunda conexión con la empresa, producen innovaciones y mantienen el impulso.

b) Los empleados no comprometidos: es decir, los que son como zombies organizacionales. Están presentes físicamente, invierten su tiempo, pero no su pasión ni su energía.

c) Los empleados activamente no comprometidos: o sea, empleados descontentos que actúan en consecuencia, demeritando el trabajo de sus compañeros (Arias, 2019).

El reporte de Gallup arroja una realidad preocupante, ya que el $79 \%$ de los colaboradores en cada empresa no están comprometidos con su trabajo. Las organizaciones de hoy están tratando de conseguir el $100 \%$ de sus resultados en un entorno altamente competitivo, lleno de amenazas y obstáculos y lo hacen con solamente el $21 \%$ de sus colaboradores comprometidos y enfocados con su puesto (Arias, 2019).

De acuerdo a un análisis sobre las tendencias en el mercado laboral en México para 2019, la remuneración económica dejó de ser el aspecto más importante para los empleados; factores como la cultura y los valores de la organización, tener retroalimentación por parte de los directivos y oportunidades de carrera se han convertido en factores de satisfacción laboral y de toma de decisiones, por lo que se espera para 2019 en el mundo laboral el empoderamiento de los empleados, la

\section{Las remuneraciones impactan positivamente en el compromiso}


transparencia en los procesos selectivos y capacitación tech, entre otros factores (Rodríguez, 2018).

Un estudio de Glassdoor en 2017 reveló que el principal indicador de satisfacción laboral no es el sueldo, sino la cultura y los valores de la organización, así como la cualidad de liderazgo de los directivos y las oportunidades de carrera (Rodríguez, 2018).

Cabe destacar, que el compromiso organizacional ha tomado interés en su estudio y análisis en las últimas décadas de tal forma que su definición se ha ido enriqueciendo desde diferentes puntos de vista estableciéndola como una variable importante para entender la naturaleza del comportamiento de los individuos en el trabajo (Villa, Marquez y Miramontes, 2016). Otros de los factores que tienen gran impacto en la fuerza laboral son las remuneraciones y el empowerment.

Por lo general, los empleados desean sistemas de compensación 0 remuneración que perciban como justos y proporcionales a sus habilidades y expectativas. El pago es una consideración importante en la administración de recursos humanos porque ofrece a los empleados una recompensa tangible por sus servicios, así como una fuente de reconocimiento y sustento (Villanueva y González, 2005).

Además, Molina (2006) señala que el término empowerment significa habilitar, conceder, permitir una iniciativa a cierta persona para actuar por sí misma y propiciar la iniciativa en otros. El empowerment es sinónimo de cultura de participación que requiere del concurso de todos para alcanzar objetivos de calidad. Este término se conforma por dos variantes: grupal e individual.

Treviño, R., Segovia, A., Alvarado, E., Guerra, P. 
De acuerdo con los antecedentes contextuales y las investigaciones empíricas relacionadas, se plantea que las remuneraciones y el empowerment psicológico en las organizaciones podrían tener un impacto positivo frente al compromiso organizacional. Aunque surgen estudios en empresas lucrativas y de servicios asistenciales, aún queda un segmento poblacional con un vacío epistemológico que en lo que se relaciona con el capital humano posee las mismas características de los hechos contextuales mencionados anteriormente: la docencia, por lo que se hace importante el presente estudio.

Pregunta de investigación

¿Cuál es el impacto de las remuneraciones en el compromiso organizacional mediante el empowerment psicológico en docentes de instituciones públicas de educación media superior?

Objetivo general

Determinar el impacto de las remuneraciones en el compromiso organizacional mediante el empowerment psicológico en docentes de instituciones públicas de educación media superior de la región citrícola del estado de Nuevo León, en México. 
Hipótesis.

$H_{1}$ : Las remuneraciones impactan positivamente en el compromiso organizacional mediante el empowerment psicológico en docentes de las instituciones públicas de educación media superior.

El estudio abarca un sujeto de estudio y área geográfica no cubierta en las investigaciones consultadas que se mencionan en la revisión de la literatura (HCNL, 2017). Ya que se han encontrado pocos estudios científicos en México, y en particular de Nuevo León, donde es probable que no se haya estudiado anteriormente en específico el modelo propuesto en este estudio que incluye el empowerment psicológico en su rol mediador. Por lo tanto, considerando los criterios del modelo de deficiencias de Creswell (2015), esta investigación contribuirá con la aportación de conocimiento en diversas brechas teóricas, ya que autores no han estudiado las variables propuestas en el contexto docente.

Entre las limitaciones de este estudio se encuentran que el diseño es transversal, ya que los datos son recabados una vez en el tiempo que lleva el estudio, no pudiendo hacer un comparativo cronológico o longitudinal para llegar a conclusiones más precisas. Otra limitación es la escasez de información en la investigación documental, con relación a las variables que se analizan en este trabajo, se encontró muy poca información al respecto en el contexto mexicano. En específico en la variable remuneraciones no se encontró literatura que ampare la relación entre ésta y el empowerment psicológico con el compromiso organizacional en este contexto.

Treviño, R., Segovia, A., Alvarado, E., Guerra, P. 


\section{Marco Teórico}

El éxito de una organización obedece, en gran medida, a las relaciones humanas, y éstas a su vez dependen de las variables actitudinales como es el caso del compromiso organizacional (CO) (MaldonadoRadillo, Guillén y Carranza, 2012), el cual está asociado a resultados organizacionales como son: el desempeño, el ausentismo, la rotación de personal, entre otros (Juaneda y González, 2007).

Jaimez y Díaz (2011) expresan que la especial atención que desde los años 50 lleva recibiendo el compromiso por parte de numerosos investigadores en el ámbito organizacional es debido a la consideración de éste como antecedente de muchos de los resultados organizacionales deseados, destacando la fuerte relación existente entre el compromiso y la decisión de abandono de la organización por parte del trabajador.

Los autores clásicos que han definido el compromiso organizacional lo han hecho desde diversas perspectivas. Desde la "perspectiva de intercambio social", el compromiso con la organización se define como el vínculo que establece el individuo con su organización, fruto de las pequeñas inversiones realizadas a lo largo del tiempo; es decir, la persona continúa en la organización porque cambiar su situación supondría sacrificar las inversiones realizadas (Becker, 1960, citado en Betanzos y Paz, 2007).

En la "perspectiva psicológica", se define como la fuerza relativa a la identificación individual e implicación con una organización en particular, y puede ser caracterizado por: (1) un fuerte deseo de

\section{Las remuneraciones impactan positivamente en el compromiso}


permanecer como miembro de una organización; (2) un acuerdo de mantener altos niveles de esfuerzo en beneficio de la organización; y (3) una creencia definitiva y aceptación de los valores y metas de la organización, que resulta de la orientación individual hacia la organización como un fin en sí mismo (Mowday, Steers, y Porter, 1979, citados en citado en Betanzos y Paz, 2007).

Desde la "perspectiva de atribución", se define el compromiso como una obligación que el individuo adquiere como resultado de realizar ciertos actos que son voluntarios, explícitos e irrevocables (Reichers, 1985, citado en Betanzos y Paz, 2007).

Son muchas las definiciones y teorías que sobre el compromiso se han enunciado históricamente, aunque quizás sea la proporcionada por Meyer y Allen (1997) una de las más desarrolladas en los últimos años (Neininger y Lehmann-Willenbrock, 2010; McInnis, Meyer y Feldman, 2009). Estos autores entendieron el compromiso como un estado psicológico que caracteriza la relación entre una persona y una organización, proponiendo un modelo tridimensional del mismo (afectivo, continuidad y normativo).

El componente afectivo del compromiso organizacional hace referencia al apego emocional del empleado con la organización, identificándose e involucrándose en la misma, mientras que el componente de continuidad se refiere al compromiso basado en el costo que el empleado asocia con irse de la organización. Por último, el componente normativo hace referencia al sentimiento de deber moral 0 gratitud que el empleado desarrolla hacia la organización como consecuencia de mejoras que ha recibido de la misma. Estas tres

Treviño, R., Segovia, A., Alvarado, E., Guerra, P. 
dimensiones funcionarían de manera independiente, aunque pueden darse algunas relaciones de aproximación (Meyer y Allen, 1997).

Barraza, Acosta y Ledezma (2008) afirman que el compromiso organizacional es, junto a liderazgo y motivación, uno de los conceptos en los que es más difícil alcanzar una definición unánime entre los distintos autores y enfoques, que satisfaga tanto a académicos como a investigadores por igual. Watson (s.f., citado en Giancola, 2010) define el compromiso del empleado como una habilidad y deseo de contribuir al éxito de la compañía. Para Lee y Kulviwat (2008), este compromiso es el grado de involucramiento y lealtad de una persona hacia la organización; mientras que Yavas y Babakus (2010) mencionan que se refiere a una actitud de adhesión entre el individuo y su empresa.

La definición clásica del compromiso organizacional es la conceptualización de Allen y Meyer (1996) quienes lo consideran como el grado con que las personas se identifican, están consustanciadas con sus organizaciones y dispuestas a continuar trabajando en ellas. Después de revisar cada una de las definiciones encontradas, se utilizará ésta para la presente investigación, siendo una de las más desarrolladas en los últimos años. Además, de acuerdo con Ríos, Rayo y Ferrer (2010) evaluar el compromiso organizacional desde los tres posibles sentimientos que experimenta un empleado (afectivo, de continuidad y normativo) brinda la oportunidad de obtener resultados más completos al definir el tipo de compromiso que experimentan y predecir su posible reacción ante determinados estímulos.

Por otro lado, las remuneraciones son consideradas como el pago que recibe el empleado por su fuerza laboral en términos

\section{Las remuneraciones impactan positivamente en el compromiso}


económicos (Rubio y Piatti, 2000). Diomede (2008) explica que las compensaciones son un sistema clave para las organizaciones siempre y cuando esté basado en la equidad. Para Villanueva y González (2005) éstas incluyen la remuneración base, los incentivos y beneficios. Para algunos autores, las remuneraciones son el motivador más importante para que la fuerza laboral se siente satisfecha y motivada, pudiéndolo relacionar con el empowerment psicológico.

Por su parte, Westerman, Beekun, Daily y Vanka (2009) identificaron la búsqueda de las organizaciones por nuevas formas de compensaciones que les permita adaptarse al mundo actual. Martín, N., Hernangómez y Martín, V. (2007) recomiendan que las compensaciones sean iguales a las aportaciones; por lo que al tener los suficientes ingresos para compensar a todos los involucrados, se alcanza una satisfacción, logrando que la organización alcance el equilibrio. Por otro lado, Segovia (2014) encontró una relación no significativa ( $b=.197$; $p=n . s$.) entre la compensación variable y el empowerment psicológico. Como se observa, es escasa o nula la existencia de artículos que relacionen en específico la variable remuneraciones con el empowerment psicológico de manera exacta o directa, e incluso con el compromiso organizacional, considerando que se realizó una búsqueda exhaustiva en diversas bases de datos como: google académico, ProQuest, Emerald, ResearchGate y Ebsco Host.

El empowerment psicológico surge a partir de las bases de Thomas y Velthouse (1990) y Spreitzer (1995) como el incremento de la motivación intrínseca en el desempeño de la función, a partir de cuatro conceptos: significado de la tarea, competencia, autodeterminación e

Treviño, R., Segovia, A., Alvarado, E., Guerra, P. 
impacto, que orientan el enfoque del individuo hacia el desempeño de su función, destacando que el empowerment psicológico no es una característica de la personalidad, más bien es un conjunto de cogniciones definido con base en determinado contexto de trabajo. Diversos estudios han relacionado el empowerment psicológico con el compromiso organizacional (Ríos et al., 2010); Chiang, Valenzuela y Lagos, 2014; Segovia, 2014).

El empowerment psicológico se utiliza como variable mediadora entre las remuneraciones y el compromiso organizacional. Por tanto, es una tercera variable, es decir, una variable interviniente que afecta la relación entre dos variables. En este caso, la variable independiente y la variable potencialmente mediadora influyen sobre la variable dependiente (Baron y Kenny, 1986; Hayes, 2013).

Al revisar la literatura, el empowerment psicológico ha sido estudiado como variable mediadora con el compromiso organizacional, tal es el caso de la investigación de Segovia (2014) cuyo modelo tenía como variables independientes el liderazgo transformacional y la compensación variable, y como variables dependientes satisfacción laboral, compromiso organizacional y trabajo en equipo dentro del constructo de resultados actitudinales. Por otra parte, Chang, Shih y Lin (2010) probaron un modelo exploratorio de empowerment en una muestra taiwanesa de enfermeras de salud escolar mediante la prueba del papel mediador del empowerment psicológico en la relación entre factores externos y actitudes relacionadas con el trabajo, específicamente la satisfacción laboral y el compromiso organizacional.

Además, en la investigación de O'Brien (2010) se observa el

\section{Las remuneraciones impactan positivamente en el compromiso}


empowerment psicológico como variable mediadora entre el empowerment estructural y el burnout en enfermeras trabajando con pacientes de centros de hemodiálisis, utilizando el análisis de correlación para probar la relación hipotética. Otros estudios han mostrado el rol mediador del empowerment psicológico entre las características estructurales del lugar de trabajo (por ejemplo, acceso a recursos e información y cultura organizacional) y resultados organizaciones positivos tales como innovación, satisfacción en el trabajo, desempeño y eficiencia (Bonias, Bartram, Leggat y Stanton, 2010; Randolph y Edward, 2011).

\section{Metodología}

El diseño metodológico utilizado fue no experimental y el tipo de investigación es correlacional/causal, con un enfoque cuantitativo, donde se utilizó la investigación bibliográfica para el desarrollo del marco teórico y la investigación de campo a través de cuestionarios para la recolección de datos para el estudio. Se elaboró un instrumento de medición válido y confiable a partir de escalas ya validadas en estudios anteriores: las encuestas que miden la efectividad de las remuneraciones en el empleado en los estudios de Madero (2012; 2010), The Psychological Empowerment Scale (Spreitzer, 1995, cit. En O'Brien, 2010) y el Cuestionario de Compromiso Organizacional de Meyer y Allen (1997), previamente traducidos y validados por expertos.

Después de elaborar el instrumento de medición adaptado al contexto de estudio, se realizó la prueba de validez de contenido bajo un análisis de expertos. Se decidió evaluar el grado de relevancia de los

Treviño, R., Segovia, A., Alvarado, E., Guerra, P. 
ítems contando con la participación de 10 expertos especialistas en Capital Humano, directivos de empresas grandes, instituciones educativas de nivel medio superior y superior y de consultoría del Estado de Nuevo León.

Se consideró una población de 296 docentes de instituciones públicas de educación media superior de la región citrícola del estado de Nuevo León, en México (UANL, 2018). Para calcular el tamaño de muestra "n" para una población finita, se utilizó la fórmula desarrollada por Gabaldon (1980) en la ecuación 1:

$$
n=\frac{N Z^{2} p q}{e^{2}(N-1)+Z^{2} p q}
$$

A partir de lo anterior, con una población $(\mathrm{N})$ de 296 docentes, un error muestral (e) de 5\%, una probabilidad de éxito (p) de 50\%, una probabilidad de fracaso (q) del $50 \%$ y un nivel de confianza del $95 \%$ ( $Z=1.96)$ se obtuvo un tamaño de muestra " $n$ " de 167 elementos. Para la recolección de datos, se llevó a cabo un muestreo no probabilístico, por cuotas, basado en dividir a la población en subgrupos exclusivos donde se debe identificar las proporciones de estos subgrupos en la población. Esto aseguró que la muestra fuera representativa de toda la población y permitió estudiar rasgos y características que se vieron en cada subgrupo, asegurando una cobertura de unidades de todos los tipos (Rabolini, 2009).

Con base en el tipo de investigación propuesto se utilizó como prueba estadística no paramétrica el modelo de ecuaciones estructurales (Structural Equation Modeling, SEM). Este modelo

\section{Las remuneraciones impactan positivamente en el compromiso}


multivariable permite examinar simultáneamente una serie de relaciones de dependencia, pero a la vez de interdependencia, ya que las variables son dependientes en una relación pero independientes en otra dentro del mismo modelo, combinando aspectos de la regresión múltiple y el análisis factorial (Leyva y Olague, 2014; Cupani, 2012). Las relaciones entre las variables latentes del SEM pueden ser de tres tipos: covarianza, efectos directos o efectos indirectos (mediadores). Ésta es una de las razones principales para utilizarlo en la presente investigación, ya que se buscó probar que el empowerment psicológico actúa en su rol mediador.

EI SEM considera dos componentes: el componente del modelo de medición (outer model) y el componente del modelo estructural (inner model). Al estar en presencia de un modelo de medición reflexivo (Valdivieso, 2016), se eligió la técnica estadística de análisis basados en la varianza o en componentes (Partial Least Squares, PLS), debido a las recomendaciones mínimas del tamaño de muestra, el objetivo orientado a la predicción, con especificaciones no paramétricas para estimar la precisión de los valores estimados y no suponiendo que los datos estaban normalmente distribuidos (Barroso, Cepeda y Roldán, 2007). Se utilizó el software SmartPLS 3.0 M3 (Hair, Hult, Ringle y Sarstedt, 2017) como herramienta estadística para el análisis de resultados.

\section{Resultados}

Con base en la aplicación del instrumento de medición a 167 docentes con las características de la población seleccionada, se presentan los resultados considerando las respuestas de los ítems en escala Likert.

Treviño, R., Segovia, A., Alvarado, E., Guerra, P. 
Cabe señalar que se comprobó la confiabilidad del instrumento mediante el Alfa de Cronbach, en donde cada una de las variables cumplieron con el criterio entre 0.7 y 0.9 considerado como aceptable.

Analizando los resultados obtenidos del personal encuestado, se encontró una mayor participación de docentes por contrato 0 eventuales (43\%). En cuanto al género se recibieron encuestas en mayor proporción del sexo femenino (51\%). La edad promedio ronda entre los 39 años, el 49\% señaló tener estudios principalmente de licenciatura, $62 \%$ son casados. Cuentan con un ingreso mensual entre $\$ 5,000$ y $\$ 9,999$ pesos y el $22 \%$ no tiene dependientes económicos, pero el $64 \%$ tiene entre 1 y 3 dependientes económicos. Aunque el $51 \%$ de los encuestados posee otro empleo, la mayoría tienen una antigüedad dentro de la institución actual de 10 años. Atienden 36 alumnos por grupo, con 23 horas-clase y tienen una experiencia laboral como docentes promedio de 13 años.

Por otra parte, al utilizar SEM para analizar el modelo, los resultados del índice de confiabilidad compuesta están dentro de los parámetros considerados como aceptables en todas las variables de esta investigación (Tabla 1). Este índice es considerado una mejor aproximación que el alfa de Cronbach, ya que supone que los parámetros son precisos y aplica sólo cuando la variable latente tiene indicadores reflexivos. En cuanto a las estadísticas de AVE (promedio de varianza extraída), según Hair et al. (2017 ) es un indicador de confiabilidad que con un valor de 0.50 o mayor indica un grado suficiente de validez convergente, lo que significa que, en promedio, la variable latente explica más de la mitad de la varianza de sus

\section{Las remuneraciones impactan positivamente en el compromiso}


indicadores. Por lo que analizando la Tabla 1, todas las variables superan el valor de 0.50 .

Tabla 1. Resultados del análisis de medición del modelo de ítems: Fiabilidad

\begin{tabular}{lccc}
\hline \multicolumn{1}{c}{ Variable } & $\begin{array}{c}\text { Alfa de } \\
\text { Cronbach }\end{array}$ & $\begin{array}{c}\text { Fiabilidad } \\
\text { compuesta }\end{array}$ & $\begin{array}{c}\text { Varianza extraída } \\
\text { media (AVE) }\end{array}$ \\
\hline $\begin{array}{l}\text { Remuneraciones } \\
\text { Empowerment }\end{array}$ & 0.936 & 0.950 & 0.759 \\
$\begin{array}{l}\text { psicológico } \\
\text { Compromiso } \\
\text { organizacional }\end{array}$ & 0.913 & 0.931 & 0.660 \\
\hline Fuente: Elaboración propia & 0.949 & 0.958 & 0.766 \\
\hline
\end{tabular}

Respecto al modelo estructural (inner model) que especifica las relaciones entre las variables no observadas o latentes. Se estiman las trayectorias o betas de las relaciones y el valor de la $\mathrm{R}^{2}$; esta última representando el grado de explicación de las variables independientes sobre la variable dependiente. Cuanto más cerca de 1 se sitúe el valor de $\mathrm{R}^{2}$, mayor será la varianza explicada por el modelo. Por lo que los valores de $R^{2}$ de 0.668 y 0.853 de la Tabla 2 , se encuentran dentro de los parámetros considerados como aceptables.

Tabla 2. Resultados de la $R^{2}$

\begin{tabular}{lll}
\hline Variable & $\mathrm{R}^{2}$ & $\mathrm{R}^{2}$ ajustada \\
\hline Empowerment psicológico & 0.668 & 0.664 \\
Compromiso organizacional & 0.853 & 0.850 \\
\hline
\end{tabular}

Fuente: Elaboración propia

Treviño, R., Segovia, A., Alvarado, E., Guerra, P. 
También se aplicó el análisis de bootstrapping no paramétrico que involucra muestras aleatorias repetidas. En la Tabla 3 se detallan los resultados obtenidos en el modelo estructural observándose las betas estandarizadas, los valores de $t$ de student y la significancia de las relaciones de la hipótesis con sus efectos directos e indirectos, siendo en este último donde se evalúa el efecto mediador de la variable empowerment psicológico.

Tabla 3. Análisis de significancia de los efectos directos e indirectos.

\begin{tabular}{|c|c|c|c|c|c|c|c|c|}
\hline $\begin{array}{l}\text { Relación } \\
\text { entre } \\
\text { variables }\end{array}$ & $\begin{array}{c}\text { Efect } \\
0 \\
\text { direct } \\
0\end{array}$ & $\begin{array}{c}t \text { de } \\
\text { stude } \\
\text { nt }\end{array}$ & $\begin{array}{c}\text { pvalu } \\
\text { e } \\
\text { (sig.) }\end{array}$ & $\begin{array}{l}\text { Significanci } \\
\text { a }(p<0.05)\end{array}$ & $\begin{array}{c}\text { Efecto } \\
\text { indirect } \\
0 \\
\text { (Con } \\
\text { EP) }\end{array}$ & $\begin{array}{c}t \text { de } \\
\text { stude } \\
\text { nt }\end{array}$ & $\begin{array}{c}\text { pvalu } \\
\text { e } \\
\text { (sig.) }\end{array}$ & $\begin{array}{c}\text { Signific } \\
\text { ancia } \\
(p<0.05 \\
\quad)\end{array}$ \\
\hline $\begin{array}{l}\text { REM } \rightarrow C \\
0\end{array}$ & 0.013 & 0.369 & 0.712 & No & 0.089 & 2.075 & 0.038 & Sí \\
\hline
\end{tabular}

Fuente: Elaboración propia. Nota: REM, remuneraciones; EP, empowerment psicológico; CO, Compromiso organizacional.

Con base en todos estos análisis estadísticos se puede concluir que los resultados muestran que la relación entre remuneraciones y compromiso organizacional tiene un efecto indirecto significativo al considerar el empowerment psicológico en su rol mediador. Esto se evalúa de acuerdo con Hair et al. (2017) como una "medicación completa"; por tanto, la hipótesis de investigación planteada se acepta (ver la Tabla 4).

\section{Las remuneraciones impactan positivamente en el compromiso}


Tabla 4. Comprobación de la hipótesis de investigación.

\begin{tabular}{|c|c|c|}
\hline Hipótesis específicas & Efecto & $\begin{array}{l}\text { Comprobación de } \\
\text { hipótesis }\end{array}$ \\
\hline \begin{tabular}{lrr} 
H1. Las & \multicolumn{2}{c}{ remuneraciones } \\
impactan positiva en el \\
compromiso & organizacional \\
mediante el & empowerment \\
psicológico & & \\
\end{tabular} & Mediación completa & Se acepta \\
\hline
\end{tabular}

Fuente: Elaboración propia.

Cabe destacar que, aunque no fue objetivo de esta investigación el estudio de los efectos directos de las remuneraciones con el compromiso organizacional, a manera comparativa los resultados comprueban que dicha relación no es significativa (ver Tabla 3).

\section{Discusión}

Los resultados muestran que se cumplió con el objetivo general de investigación de determinar el impacto de las remuneraciones en el compromiso organizacional considerando al empowerment psicológico en su rol mediador. De acuerdo con la interpretación del análisis estadístico, no se rechaza la hipótesis de investigación (H1), ya que los resultados avalan la relación positiva de las remuneraciones con el compromiso organizacional mediante el empowerment psicológico. Cabe señalar que aunque no se encontró un número importante de teorías ni investigaciones empíricas que estudiaran dichas variables como tal, es decir, en la forma del modelo propuesto en este estudio, el trabajo brinda un aporte al vacío epistemológico existente en este

Treviño, R., Segovia, A., Alvarado, E., Guerra, P. 
campo de la ciencia y en el segmento poblacional estudiado y se destacan algunas similitudes de manera parcial con lo investigado por autores que analizaron alguna de las variables de manera independiente, no como el modelo de mediación completo propuesto.

Respecto a la relación positiva individual del empowerment psicológico y el compromiso organizacional, muestra similitud con lo encontrado en pequeñas y medianas empresas de Celaya, Guanajuato por Ríos et al. (2010), quienes explican también que dicha relación ha sido un campo poco explorado desde la perspectiva organizacional, por lo que ellos utilizaron un modelo generado con base en las dimensiones de ambas variables.

Además, los resultados coinciden con lo que Jáimez y Díaz (2011) encontraron en trabajadores de una empresa española donde el empowerment psicológico muestra una relación de causalidad con el compromiso afectivo, y no con las otras 2 dimensiones del compromiso organizacional. Mientras que otro estudio de Chiang et al. (2014) donde utilizaron un modelo de regresión en 219 trabajadores de pequeñas y medianas empresas en Chile, obtuvieron que el compromiso organizacional se explica por las dimensiones de impacto, autodeterminación y significado del empowerment psicológico en las empresas medianas, no siendo así para las pequeñas.

La relación parcial entre empowerment psicológico y el compromiso organizacional, coincide también con lo encontrado por Chiang et al. (2014) y Segovia (2014). Sin embargo, Segovia (2014) al estudiar la variable compensación variable con empowerment

\section{Las remuneraciones impactan positivamente en el compromiso}


psicológico, rechazó su hipótesis al encontrar una relación no significativa.

Finalmente en el presente trabajo se encontró una relación inexistente dentro de los efectos directos entre las remuneraciones y el compromiso organizacional (es decir, no considerando el empowerment psicológico en su rol mediador), lo que significa que cuanto más reciben los docentes de remuneraciones de las instituciones educativas, éstos no generan un mayor compromiso organizacional. Esto coincide con lo que se plantea en los antecedentes contextuales de la presente investigación, que en la época actual en México, la remuneración económica dejó de ser el aspecto más importante para los empleados; factores como la cultura y los valores de la organización, tener retroalimentación por parte de los directivos y oportunidades de carrera se han convertido en factores de satisfacción laboral y de toma de decisiones, todo ello traducido en el empoderamiento de los empleados, conocido mejor como empowerment psicológico (Rodríguez, 2018).

\section{Conclusiones}

Los hallazgos muestran que el rol mediador del empowement psicológico es significativo en la relación de las remuneraciones con el compromiso organizacional. Pero en cuanto a los efectos directos de dicha relación, los docentes señalan que mayores remuneraciones no implican un mayor grado de compromiso organizacional. Lo anterior refleja que la búsqueda de bienestar y empoderamiento de los docentes en el lugar de trabajo se manifiesta en esa relación positiva donde la

Treviño, R., Segovia, A., Alvarado, E., Guerra, P. 
organización es capaz de estimular su empowerment frente a las necesidades educativas y laborales, incrementando el compromiso organizacional de los empleados hacia la institución.

Dentro de las contribuciones de este trabajo y de acuerdo a los criterios del modelo de deficiencias de Creswell (2015), se hacen aportaciones importantes al vacío epistemológico en el contexto mexicano, tanto en la población y área seleccionada en el presente estudio.

Esta investigación permite a las instituciones educativas diseñar estrategias, con base en las remuneraciones pero sustentadas por motivaciones intrínsecas como el empowerment psicológico, para que los docentes se sientan apoyados en múltiples aspectos, trayendo consigo un incremento en el compromiso hacia su área laboral. Sobre todo que se considera que es una de las profesiones con mayor carga laboral dentro y fuera de las aulas, siendo imprescindible el grado bienestar que los profesores tienen en sus instituciones y la forma en que éstas los apoyan.

Entre las acciones propuestas para las instituciones, el sistema educativo en México y otros contextos, se destacan las siguientes:

1. Implementar y/o incrementar programas de estímulos económicos mediante evaluación que correlacionen los resultados de estudiantes con el esfuerzo y compromiso del docente.

2. Implementar programas de capacitación que incidan en las actitudes y conductas de los empleados, no sólo en sus áreas disciplinares.

\section{Las remuneraciones impactan positivamente en el compromiso}


3. Implementar programas de atención para los docentes que presenten bajo nivel de compromiso a través de revisiones médico-psicológicas, encuestas o entrevistas laborales.

4. Asegurar que los profesores tengan derecho a una formación continua relevante y pertinente, enfocada en el desarrollo integral y en los aprendizajes de los estudiantes, buscando apoyos económicos 0 incentivos para los docentes durante dicho proceso.

5. Establecer estándares y criterios de evaluación válidos, confiables y coherentes para ser aplicados con pertinencia que brinden información fehaciente sobre la satisfacción laboral y compromiso de los docentes, así como su correlación con el apoyo que sus instituciones les brindan.

6. Enfocar la formación, el apoyo, recursos y oportunidades de desarrollo de los docentes de manera que impacte directamente en las prácticas de enseñanza y el desarrollo de los estudiantes, así como en generar docentes más comprometidos y satisfechos.

7. De acuerdo con su antigüedad y/o desempeño, promover que los docentes participen en diversos proyectos de gestión institucional, así como la asignación de responsabilidades de mayor grado, procurando que la alta participación de los docentes en la gestión escolar esté altamente relacionada con buenos niveles de bienestar social.

Treviño, R., Segovia, A., Alvarado, E., Guerra, P. 
8. Mejorar el apoyo económico y en oportunidades de desarrollo brindadas al docente para realizar su trabajo y asegurar el aprovechamiento de los alumnos y alumnas; disminuyendo el ausentismo, elevando la eficiencia terminal y reduciendo las inequidades del sistema educativo.

9. Determinar la combinación específica de incentivos monetarios y no monetarios que sean más efectivos. Dejando de lado los reconocimientos o consecuencias relativos al resultado, sino que para ser considerados maestros eficientes, los estudiantes deben demostrar niveles de aprovechamiento satisfactorios.

10. Promover mecanismos de atención laboral que mejoren el clima laboral, el comportamiento organizacional y los resultados en las instituciones educativas.

De igual manera, este trabajo de investigación tiene aplicaciones no sólo para las instituciones sujetas a estudio, sino para las instituciones de otras entidades del país, dependencias de otros niveles del sistema educativo $y$, finalmente, para organizaciones de cualquier otro giro que se interesen por los factores que verdaderamente incidan para tener empleados más competitivos y comprometidos, cuyos resultados trasciendan en dichas entidades.

Ya que no fue parte del objetivo de investigación analizar a la muestra por estratos, se recomienda y se planea continuar con la investigación en este contexto considerando las características de los

\section{Las remuneraciones impactan positivamente en el compromiso}


docentes (género, estado civil, escolaridad, ingresos, etc.), lo que generaría más aportaciones al estudio.

\section{Referencias}

Allen, N. y Meyer, J. (1996). Affective, continuance and normative commitment to the organization: An examination of construct validity. Journal of Vocational Behavior, 49, 252-276

Arias, A. (2019). Compromiso. El horizonte. Recuperado el 25 de febrero de 2019, de http://www.elhorizonte.mx/opinion/editorial/compromiso/2444513

Baron, R. M. y Kenny, D. A. (1986). The moderator-mediator variable distinction in social psychological research: Conceptual, strategic and statistical considerations. Journal of Personality and Social Psychology, 51, 1173-1182

Barraza, M. A., Acosta CH. M. y Ledesma, M. Z. E. (2008). Compromiso organizacional de los docentes de una institución de Educación Media Superior. Recuperado el 15 de febrero de 2016, de http://www.comie.org.mx/congreso/memoriaelectronica/v10/pdf/area_tematica_ 16/ponencias/0414-F.pdf

Barroso, C., Cepeda, G., \& Roldán, J. L. (2007). Investigar en economía de la empresa: ¿partial least squares o modelos basados en la covarianza?. In EI comportamiento de la empresa ante entornos dinámicos: XIX Congreso anual y XV Congreso Hispano Francés de AEDEM (p. 63). Asociación Española de Dirección y Economía de la Empresa (AEDEM)

Betanzos, N., y Paz, F. (2007). Análisis psicométrico del compromiso organizacional como variable actitudinal. Anales de psicología, 23(2), 207-215

Bonias, D., Bartram, T., Leggat, S., y Stanton, P. (2010). Does psychological empowerment mediate the relationship between high performance work systems and patient care quality in hospitals? Asia Pacific Journal of Human Resources, 48(3), 319-337. http://dx.doi.org/10.1177/103844111110381667

Treviño, R., Segovia, A., Alvarado, E., Guerra, P. 
Calderón, J., Laca, F., Pando, M. \& Pedroza, F. (2015). Relación de la socialización organizacional y el compromiso organizacional en trabajadores mexicanos. Psicogente, 18(34), 267-277. http://doi.org/10.17081/psico.18.34.503

Chang, L. C., Shih, C. H., y Lin, S. M. (2010). The mediating role of psychological empowerment on job satisfaction and organizational commitment for school health nurses: A cross-sectional questionnaire survey. International Journal of Nursing Studies, 47(4), 427-433

Chiang, M.M., Valenzuela, L. y Lagos, M.A. (2014). RELATIONSHIP BETWEEN PSYCHOLOGICAL EMPOWERMENT AND ORGANIZATIONAL COMMITMENT IN SMALL AND MEDIUM ENTERPRISES. Multidisciplinary Business Review, 7 (1), (Diciembre 2014)

CNNExansión. (2016). 5 beneficios de negocio que trae el "engagement" laboral. La competitividad de las empresas se eleva cuando sus colaboradores se comprometen emocionalmente con la organización. Recuperado el 10 de noviembre de 2016, de http://expansion.mx/carrera/2016/08/10/5-beneficiosde-negocio-que-trae-el-engagement-laboral

Creswell, J. W. (2015). Research Design: Qualitative, quantitative, and mixed method approaches. 4th. Edition. Thousand Oaks: Sage Publications

Cupani, M. (2012). Análisis de Ecuaciones Estructurales: conceptos, etapas de desarrollo y un ejemplo de aplicación. REVISTA TESIS Facultad De Psicología, 2(1), 186-199

Deloitte (2016). Encuesta Millennials 2016 Conquistando a la siguiente generación de líderes. Recuperado el 15 de septiembre de 2016 de https://www2.deloitte.com/mx/es/pages/aboutdeloitte/articles/millenial2016.html

Deloitte. (2017). Encuesta Millennials 2017. ¿Qué mundo quieren habitar? Recuperado el 28 de mayo de 2018, de https://www2.deloitte.com/mx/es/pages/aboutdeloitte/articles/encuesta-millennial-2017.html

Diomede, H. (2008). Compensaciones se escribe con "E" de equidad. Recuperado el 18 de octubre de 2015, de

\section{Las remuneraciones impactan positivamente en el compromiso}


http://pt.slideshare.net/Diomede07/compensaciones-se-escribe-con-e-deequidad

Gabaldon, N. (1980). Algunos conceptos de muestreo (3ra. Ed.). Caracas, Venezuela: Universidad Central de Venezuela, Facultad de Ciencias Económicas y Sociales, División de publicaciones

Giancola, F. (2010). Common misconceptions in employee rewards management. Benefits \& Compensation Digest, 47(9), 34-38

Hair, J. F., Hult, G. T. M., Ringle, C. M., y Sarstedt, M. (2017). A Primer on Partial Least Squares Structural Equation Modeling. 2nd Ed. Thousand Oaks: Sage

Hayes, A. F. (2013). Introduction to Mediation, Moderation, and Conditional Process Analysis: A Regression-Based Approach. New York, NY: The Guilford Press

HCNL. (2017). Ley de Fomento a la Inversión y al Empleo para el Estado de Nuevo León. Recuperado el 20 de febrero de 2018, de http://www.hcnl.gob.mx/trabajo_legislativo/leyes/leyes/ley_de_fomento_a_la_in version_y_al_empleo_para_el_estado_de_nuevo_leon/

Jaimez, M. J. y Díaz, F. (2011). El empowerment organizacional: el inicio de una gestión saludable en el trabajo. Revista de Trabajo y Seguridad Social. Recursos Humanos, 344, 209-232

Juaneda, E. y González, L. (2007). Definición, antecedentes y consecuencias del compromiso organizativo. En Conocimiento, innovación y emprendedores: Camino al futuro, ISBN 84-690-3573-8

Lee, K., y Kulviwat, S. (2008). Korean workers' motivation tools: Commitment and incentive-based motivation and their relative impact on behavioral work outcome. Multinational Business Review, 16(4), 87-109

Leyva, O., y Olague, J. T. (2014). Modelo de ecuaciones estructurales por el método de mínimos cuadrados parciales (Partial Least Squares-PLS), en Sáenz López, J \& Tamez González, G (Coord). Métodos y técnicas cualitativas y cuantiativas aplicables a las investigaciones sociales. México, D.F. Tirant Humanidades

Madero, S. M. (2012). La efectividad de las compensaciones, la satisfacción del trabajador y las dimensiones del ambiente laboral. CIENCIA-UANL, 15(57), 93100

Treviño, R., Segovia, A., Alvarado, E., Guerra, P. 
Madero, S. M. (2010). Factores relevantes del desarrollo profesional y de compensaciones en la carrera laboral del trabajador. Contaduría y administración, (232), 109-130

Maldonado-Radillo, S.E., Guillén, A.M. y Carranza, R.E. (2012). El compromiso organizacional del personal docente y administrativos de una Universidad Pública. En Revista Internacional Administración \&Finanzas, 5 (4), 135-142

Martín, N., Hernángomez, J., y Martín, V. (2007). El deleite de la eficiencia. Universia Business Review, 5(14), 56-67

Mclnnis, Meyer y Feldman (2009). Psychological contracts and their implications for commitment: A feature-based approach. Journal of Vocational Behavior, 74, 165-180

Meyer, J.P. y Allen, N.J. (1997).Commitment in the workplace: Theory, research and application. Thousand Oaks:CA: Sage

Molina, R. (2006). Empowerment ¿actitud mental?, ¿técnica?, ¿estrategia? 0 ¿moda? México: Trillas

Neininger, A. y Lehmann-Willenbrock (2010). Effects of team and organizational commitment- A longitudinal study. Journal of vocational behaviour, 76, 567-579

O'Brien, J. L. (2010). Structural empowerment, psychological empowerment and burnout in registered staff nurses working in outpatient dialysis centers (Doctoral dissertation, Rutgers University-Graduate School-Newark)

Rabolini, N. M. (2009). Técnicas de muestreo y determinación del tamaño de la muestra en investigación cuantitativa. Revista argentina de humanidades y ciencias sociales, 2

Randolph, W. y Edward, R. (2011). Managerial use of power bases in a model of managerial empowerment practices and employee psychological empowerment. Journal of Leadership \& Organizational Studies, 18(1), 95106.http://dx.doi.org/10.1177/1548051810379798

Ríos, M., Rayo, M., y Ferrer, J. (2010). El empowerment como predictor del compromiso organizacional en las Pymes. Contaduría y administración, 26(231), 103-125

\section{Las remuneraciones impactan positivamente en el compromiso}


Rodríguez, K. (2018) Tendencias en el mercado laboral en México para 2019. Recuperado el 25 de febrero de 2019, de http://computerworldmexico.com.mx/tendencias-en-el-mercado-laboral-enmexico-para-2019/

Rubio, V. y Piatti, G.M. (2000).Manual de remuneraciones. Argentina: Cuyo

Segovia, A. (2014). El liderazgo, la compensación variable, el empowerment psicológico y su impacto en la efectividad del empleado: un enfoque de modelación mediante ecuaciones estructurales (Doctoral dissertation, Universidad Autónoma de Nuevo León)

Spreitzer, G. M. (1995). "Psychological Empowerment in the Workplace: Dimensions, Measurement, and Validation". The Academy of Management Journal, 38(5), 1442-1465

Thomas, K. y Velthouse, B. (1990). Cognitive Elements of Empowerment: An "Interpretive" Model of Intrinsic Task Motivation. Academy of Management Review, 15, 666-681. Recuperado el 28 de abril de 2018, de https://www.jstor.org/stable/258687?seq=1\#page_scan_tab_contents. DOI: $10.2307 / 258687$

Universidad Autónoma de Nuevo León. (2018). Directorio de planteles de nivel medio superior (Nuevo León). Proceso de Asignación de Espacios en la Educación Media Superior del Estado de Nuevo León 2018. Recuperado el 28 de abril de 2018, de http://www.uanl.mx/alumnos/convocatorias/registro-de-aspirantespreparatoria-en-modalidad-presencial.html

Valdivieso, C.E. (2016). Comparación de los modelos formativo, reflexivo y de antecedentes de evaluación estudiantil del servicio de docencia//Comparison of Formative, Reflective, and Antecedents Models of Students Evaluation of Teaching Service. Revista de Métodos Cuantitativos para la Economía y la Empresa, 16, 95-120

Villa, O., y Marquez, Miramontes, B. L. (2016). Relación del empowerment con el compromiso organizacional influenciado por el capital humano y prácticas de alto involucramiento de empleados en maquiladoras automotrices. In Global

Treviño, R., Segovia, A., Alvarado, E., Guerra, P. 
Conference on Business \& Finance Proceedings (Vol. 11, No. 1, p. 855). Institute for Business \& Finance Research

Villanueva, A. y González, E. (2005). Gestión en las compensaciones. Chile: Paidós

Westerman, J.W., Beekun, R.I., Daily, J., y Vanka, S. (2009). Personality and national culture. Predictors of compensation strategy preferences in the USA and India. Management Research News, 32(8), 767-781

Yavas, U., y Babakus, E. (2010). Relation between organizational support, customer orientation and work outcomes. The International Journal of Bank Markting, 28(3), 222-238 